\title{
Exploring the effect of Lorentz invariance violation with the currently running long-baseline experiments
}

\author{
Rudra Majhi ${ }^{1, a}$, Soumya Chembra ${ }^{2, b}$, Rukmani Mohanta ${ }^{1, c}$ \\ ${ }^{1}$ School of Physics, University of Hyderabad, Hyderabad 500046, India \\ 2 Institute of Physics, Sachivalaya Marg, Sainik School Post, Bhubaneswar 751005, India
}

Received: 10 December 2019 / Accepted: 24 April 2020 / Published online: 6 May 2020

(C) The Author(s) 2020

\begin{abstract}
Neutrinos are the fundamental particles, blind to all kind of interactions except the weak and gravitational. Hence, they can propagate very long distances without any deviation. This characteristic property can thus provide an ideal platform to investigate Planck suppressed physics through their long distance propagation. In this work, we intend to investigate CPT violation through Lorentz invariance violation (LIV) in the long-baseline accelerator based neutrino experiments. Considering the simplest four-dimensional Lorentz violating parameters, for the first time, we obtain the sensitivity limits on the LIV parameters from the currently running long-baseline experiments $\mathrm{T} 2 \mathrm{~K}$ and NOvA. In addition to this, we show their effects on mass hierarchy and $\mathrm{CP}$ violation sensitivities by considering NOvA as a case study. We find that the sensitivity limits on LIV parameters obtained from $\mathrm{T} 2 \mathrm{~K}$ are much weaker than that of $\mathrm{NO} v \mathrm{~A}$ and the synergy of $\mathrm{T} 2 \mathrm{~K}$ and $\mathrm{NO} \nu \mathrm{A}$ can improve these sensitivities. All these limits are slightly weaker ( $2 \sigma$ level) compared to the values extracted from Super-Kamiokande experiment with atmospheric neutrinos. Moreover, we observe that the mass hierarchy and CPV sensitivities are either enhanced or deteriorated significantly in the presence of LIV as these sensitivities crucially depend on the new CP-violating phases. We also present the correlation between $\sin ^{2} \theta_{23}$ and the LIV parameter $\left|a_{\alpha \beta}\right|$, as well as $\delta_{C P}$ and $\left|a_{\alpha \beta}\right|$.
\end{abstract}

\section{Introduction}

Neutrinos are considered to be the most fascinating particles in nature, posses many unique and interesting features in contrast to the other Standard Model (SM) fermions. The effort

\footnotetext{
a e-mail: rudra.majhi95@gmail.com

b e-mail: soumyac20@gmail.com

c e-mail: rmsp@uohyd.ac.in (corresponding author)
}

of many dedicated neutrino oscillation experiments [1-16] over the last two decades, provide us a splendid understanding about the main features of these tiny and elusive particles. Indeed we now know that neutrinos are massive albeit extremely light, and change their flavour as they propagate. This intriguing characteristic, known as neutrino oscillation, bestows the first experimental evidence of physics beyond the SM. Without the loss of generality, SM is considered as a low-energy effective theory, emanating from a fundamental unified picture of gravity and quantum physics at the Planck scale. To understand the nature of the Plank scale physics through experimental signatures is therefore of great importance, though extremely challenging to identify. Lorentz symmetry violation constitutes one of such signals, basically associated with tiny deviation from relativity. In recent times, the search for Lorentz violating and related CPT violating signals have been explored over a wide range of systems and at remarkable sensitivities [17-28]. One of the phenomenological consequences of CPT invariance is that a particle and its anti-particle will have exactly the same mass and lifetime and if any difference observed either in their mass or lifetime, would be a clear hint for CPT violation. There exists stringent experimental bounds on Lorentz and CPT violating parameters from kaon and the lepton sectors. For the kaon system, the observed mass difference provides the upper limit on CPT violation as $\left|m_{K^{0}}-m \overline{K^{0}}\right| / m_{K}<6 \times 10^{-18}$ [29], which is quite stringent. However, parametrizing in terms of $m_{K}^{2}$ rather than $m_{K}$, as kaon is a boson and the natural mass parameter appears in the Lagrangian is the squared mass, the kaon constraint turns out to be $\left|m_{K^{0}}^{2}-m \frac{2}{K^{0}}\right|<0.25 \mathrm{eV}^{2}$, which is comparable to the bounds obtained from neutrino sector, though relatively weak. Furthermore, neutrinos are fundamental particles, unlike the kaons hence, the neutrino system can be regarded as a better probe to search for CPT violation. For example, the current neutrino oscillation data provides the most stringent bounds: $\left|\Delta m_{21}^{2}-\Delta \bar{m}_{21}^{2}\right|<5.9 \times 10^{-5} \mathrm{eV}^{2}$ 
and $\left|\Delta m_{31}^{2}-\Delta \bar{m}_{31}^{2}\right|<1.1 \times 10^{-3} \mathrm{eV}^{2}$ [30]. Recently, MINOS experiment [31] has also provided the bound on the atmospheric mass splitting for the neutrino and antineutrino modes at $3 \sigma$ C.L. as $\left|\Delta m_{31}^{2}-\Delta \bar{m}_{31}^{2}\right|<0.8 \times 10^{-3} \mathrm{eV}^{2}$. If these differences are due to the interplay of some kind of CPT violating new physics effects, they would influence the oscillation phenomena for neutrinos and antineutrinos as well as have other phenomenological consequences, such as neutrino-antineutrino oscillation, baryogenesis [32] etc.

It is well known that the local relativistic quantum field theories are based on three main ingredients: Lorentz invariance, locality and hermiticity. The CPT violation is intimately related to Lorentz violation, as possible CPT violation can arise from Lorentz violation, non-locality, non-commutative geometry etc. So if CPT violation exists in nature and is related to quantum gravity, which is supposedly non-local and expected to be highly suppressed, long-baseline experiments have the capability to probe such effects. Here, we present a brief illustration about, how the violation of Lorentz symmetry can affect the neutrino propagation. In general, Lorentz symmetry breaking and quantum gravity are interrelated, which requires the existence of a universal length scale for all frames. However, such universal scale is in conflict with general relativity, as length contraction is one of the consequences of Lorentz transformation. Such contradiction can be avoided by the modification of Lorentz transformations (or in other words modifying dispersion relations). The effects of perturbative Lorentz and CPT violation on neutrino oscillations has been studied in [33]. Moreover, it has been shown explicitly in Ref. [34], how the oscillation probability gets affected by the modified dispersion relation, however, for the sake of completeness we will present a brief discussion about it. The modified energy-momentum relation for the neutrinos can be expressed as

$E_{i}^{2}=p_{i}^{2}+\frac{1}{2} m_{i}^{2}\left(1+e^{2 A_{i} E_{i} / m_{i}^{2}}\right)$,

where $m_{i}, E_{i}$ and $p_{i}$ are the mass, energy and momentum of the $i$ th neutrino in the mass basis, and $A_{i}$ is the dimensionful and Lorentz symmetry breaking parameter. Assuming that all the neutrinos have the same energy $(E)$, the probability of transition from a given flavour $\alpha$ to another flavour $\beta$ for two neutrino case is given as

$P\left(v_{\alpha} \rightarrow v_{\beta}\right)=1-\sin ^{2} 2 \theta \sin ^{2}\left(\frac{\Delta p L}{2}\right)$,

where $\theta$ represents the mixing angle and

$\Delta p \approx \frac{\Delta m^{2}}{2 E}+\frac{1}{2}\left(A_{i}-A_{j}\right)$,

with $\Delta m^{2}=m_{i}^{2}-m_{j}^{2}$. Hence, the neutrino oscillation experiments might provide the opportunity to test this kind of new physics. The limits on Lorentz and CPT violating parameters from MINOS experiment are presented in [35]. The possible effect of Lorentz violation in neutrino oscillation phenomena has been intensely investigated in recent years $[21,33,34,36-$ 54].

In this paper, we are interested to study the phenomenological consequences introduced in the neutrino sector due to the presence of Lorentz invariance violation terms. In particular, we investigate the impact of such new contributions on the neutrino oscillation probabilities for $\mathrm{NO} v \mathrm{~A}$ experiment. Further, we obtain the sensitivity limits on the LIV parameters from the currently running long-baseline experiments $\mathrm{T} 2 \mathrm{~K}$ and $\mathrm{NO} v \mathrm{~A}$. We also investigate the implications of LIV effects on the determination of mass ordering as well as the $\mathrm{CP}$ violation discovery potential of $\mathrm{NO} v \mathrm{~A}$ experiment.

The outline of the paper is as follows. In Sect. 2, we present a brief discussion on the theoretical framework for incorporating LIV effects and their implications on neutrino oscillation physics. The simulation details used in this analysis are discussed in Sect. 3. The impact of LIV parameters on the $v_{\mu} \rightarrow v_{e}$ oscillation probability is presented in Sect. 4. Section 5 contains the discussion on the sensitivity limits on LIV parameters, which can be extracted from T2K and NOvA experiments. The discussion on how the discovery potential for $\mathrm{CP}$ violation and the mass hierarchy sensitivity get affected due to the presence of LIV, the correlation between LIV parameters and $\delta_{C P}$ as well as $\theta_{23}$ are illustrated in Sect. 6. Finally we present our summary in Sect. 7.

\section{Theoretical framework}

The Lorentz invariance violation effect can be introduced as a small perturbation to the standard physics descriptions of neutrino oscillations. Thus, the effective Lagrangian that describes Lorentz violating neutrinos and anti-neutrinos [17, 55 ] is given as

$\mathcal{L}=\frac{1}{2} \bar{\Psi}_{A}\left(i \gamma^{\mu} \partial_{\mu} \delta_{A B}-M_{A B}+\hat{\mathcal{Q}}_{A B}\right) \Psi_{B}+$ h.c.,

where $\Psi_{A(B)}$ is a $2 N$ dimensional spinor containing the spinor field $\psi_{\alpha(\beta)}$ with $\alpha(\beta)$ ranges over $N$ spinor flavours and their charge conjugates $\psi_{\alpha(\beta)}^{C}=C \bar{\psi}_{\alpha(\beta)}^{T}$, expressed as $\Psi_{A(B)}=\left(\psi_{\alpha(\beta)}, \psi_{\alpha(\beta)}^{C}\right)^{T}$ and the Lorentz violating operator is characterized by $\hat{\mathcal{Q}}$. Restricting ourselves to only a renormalizable theory (incorporating terms with mass dimension $\leq 4$ ), one can symbolically write the Lagrangian density for neutrinos as [55]

$$
\begin{aligned}
\mathcal{L}_{\mathrm{LIV}}= & -\frac{1}{2}\left[p_{\alpha \beta}^{\mu} \bar{\psi}_{\alpha} \gamma_{\mu} \psi_{\beta}+q_{\alpha \beta}^{\mu} \bar{\psi}_{\alpha} \gamma_{5} \gamma_{\mu} \psi_{\beta}\right. \\
& \left.-i r_{\alpha \beta}^{\mu \nu} \bar{\psi}_{\alpha} \gamma_{\mu} \partial_{\nu} \psi_{\beta}-i s_{\alpha \beta}^{\mu \nu} \bar{\psi}_{\alpha} \gamma_{5} \gamma_{\mu} \partial_{\nu} \psi_{\beta}\right]+ \text { h.c. }
\end{aligned}
$$


where $p_{\alpha \beta}^{\mu}, q_{\alpha \beta}^{\mu}, r_{\alpha \beta}^{\mu \nu}$ and $s_{\alpha \beta}^{\mu \nu}$ are the Lorentz violating parameters, in the flavor basis. Since, only left-handed neutrinos are present in the SM, the observable effects which can be explored in the neutrino oscillation experiments can be parametrized as

$\left(a_{L}\right)_{\alpha \beta}^{\mu}=(p+q)_{\alpha \beta}^{\mu}, \quad\left(c_{L}\right)_{\alpha \beta}^{\mu \nu}=(r+s)_{\alpha \beta}^{\mu \nu}$.

These parameters are hermitian matrices in the flavour space and can affect the standard vacuum Hamiltonian. The parameter $\left(a_{L}\right)_{\alpha \beta}^{\mu}$ is related to CPT violating neutrinos and $\left(c_{L}\right)_{\alpha \beta}^{\mu \nu}$ is associated with CPT-even, Lorentz violating neutrinos. Here, we consider the isotropic model (direction-independent) for simplicity, which appears when only the time-components of the coefficients are non-zero i.e., terms with $\mu=\nu=0$ [17]. The sun-centred isotropic model is a popular choice and in this frame, the Lorentz-violating isotropic terms are considered as $(a)_{\alpha \beta}^{0}$ and $(c)_{\alpha \beta}^{00}$. Here onwards we change the notation $\left(a_{L}\right)_{\alpha \beta}^{0}$ to $a_{\alpha \beta}$ and $\left(c_{L}\right)_{\alpha \beta}^{00}$ to $c_{\alpha \beta}$ for convenience. Taking into account only these isotropic terms of Lorentz violation parameters, the Hamiltonian for neutrinos, including LIV contributions becomes

$H=H_{\mathrm{vac}}+H_{\mathrm{mat}}+H_{\mathrm{LIV}}$,

where $H_{\mathrm{vac}}$ and $H_{\text {mat }}$ correspond to the Hamiltonians in vacuum and in the presence of matter effects and $H_{\text {LIV }}$ refers to the LIV Hamiltonian. These are expressed as

$$
\begin{aligned}
H_{\mathrm{vac}} & =\frac{1}{2 E} U\left(\begin{array}{ccc}
m_{1}^{2} & 0 & 0 \\
0 & m_{2}^{2} & 0 \\
0 & 0 & m_{3}^{2}
\end{array}\right) U^{\dagger}, \\
H_{\mathrm{mat}} & =\sqrt{2} G_{F} N_{e}\left(\begin{array}{lll}
1 & 0 & 0 \\
0 & 0 & 0 \\
0 & 0 & 0
\end{array}\right), \\
H_{\mathrm{LIV}} & =\left(\begin{array}{lll}
a_{e e} & a_{e \mu} & a_{e \tau} \\
a_{e \mu}^{*} & a_{\mu \mu} & a_{\mu \tau} \\
a_{e \tau}^{*} & a_{\mu \tau}^{*} & a_{\tau \tau}
\end{array}\right)-\frac{4}{3} E\left(\begin{array}{lll}
c_{e e} & c_{e \mu} & c_{e \tau} \\
c_{e \mu}^{*} & c_{\mu \mu} & c_{\mu \tau} \\
c_{e \tau}^{*} & c_{\mu \tau}^{*} & c_{\tau \tau}
\end{array}\right),
\end{aligned}
$$

where $U$ is the neutrino mixing matrix, $G_{F}$ is the Fermi constant and $N_{e}$ is the number density of electrons. The factor $-4 / 3$ in $H_{\text {LIV }}$ arises from the non-observability of the Minkowski trace of the CPT-even LIV parameter $c_{L}$, which forces the $x x, y y$, and $z z$ components to be related to the 00 component [17]. Since the mass dimensions of $a_{\alpha \beta}$ and $c_{\alpha \beta}$ LIV parameters are different, the effect of $a_{\alpha \beta}$ is proportional to the baseline $L$, whereas $c_{\alpha \beta}$ is proportional to $L E$ and in this work we focus only on the impact of $a_{\alpha \beta}$ parameters on the physics potential of currently running long-baseline experiments NOvA and T2K. Another possible way to introduce an isotropic Lorentz invariance violation is by considering the modified dispersion relation (MDR) preserving rotational symmetry [56], which can be expressed as

$$
E^{2}-\left(1-f\left(\frac{|\vec{p}|}{E}\right)\right)|\vec{p}|^{2}=m^{2}
$$

where the perturbative function $f$ preserves the rotational invariance. However, this approach is not adopted in this work.

It should be noted that, the Hamiltonian in the presence of LIV (7), is analogous to that in the presence of NSI in propagation, which is expressed as [57]

$H=H_{\mathrm{vac}}+H_{\mathrm{mat}}+H_{\mathrm{NSI}}$,

with

$H_{\mathrm{NSI}}=\sqrt{2} G_{F} N_{e}\left(\begin{array}{ccc}\epsilon_{e e}^{m} & \epsilon_{e \mu}^{m} & \epsilon_{e \tau}^{m} \\ \epsilon_{\mu e}^{m} & \epsilon_{\mu \mu}^{m} & \epsilon_{\mu \tau}^{m} \\ \epsilon_{\tau e}^{m} & \epsilon_{\tau \mu}^{m} & \epsilon_{\tau \tau}^{m}\end{array}\right)$,

where $\epsilon_{\alpha \beta}^{m}$ characterizes the relative strength between the matter effect due to NSI and the standard scenario. Thus, one obtains a correlation between the NSI and CPT violating scenarios through

$a_{\alpha \beta}=\sqrt{2} G_{F} N_{e} \epsilon_{\alpha \beta}^{m} \equiv V_{C C} \epsilon_{\alpha \beta}^{m}$,

where $V_{C C}=\sqrt{2} G_{F} N_{e}$. The off-diagonal elements of the CPT violating LIV Hamiltonian $\left(a_{e \mu}, a_{e \tau}\right.$ and $\left.a_{\mu \tau}\right)$ are the lepton flavor violating LIV parameters, which can affect the neutrino flavour transition, are our subject of interest. These parameters are expected to be highly suppressed and the current limits on their values (in $\mathrm{GeV}$ ), which are constrained by Super-Kamikande atmoshperic neutrinos data at $95 \%$ C.L. [44] as

$$
\begin{aligned}
& \left|a_{e \mu}\right|<2.5 \times 10^{-23}, \quad\left|a_{e \tau}\right|<5 \times 10^{-23}, \\
& \left|a_{\mu \tau}\right|<8.3 \times 10^{-24} .
\end{aligned}
$$

\section{Simulation details}

In this section, we briefly describe the experimental features of $\mathrm{T} 2 \mathrm{~K}$ and NOvA experiments that we consider in the analysis.

$\mathrm{NO} v \mathrm{~A}$ is a currently running long-baseline accelerator experiment, with two totally active scintillator detectors, Near Detector (ND) and Far Detector (FD). ND is placed at around $1 \mathrm{~km}$ and FD is at a distance of $810 \mathrm{~km}$ away from source and both the detectors are off-axial by $14.6 \mathrm{mrad}$ in nature, which provides a large flux of neutrinos at an energy of $2 \mathrm{GeV}$, the energy at which oscillation from $v_{\mu}$ to $v_{e}$ is expected to be at a maximum. It uses very high intensity $v_{\mu}$ beam, coming from NuMI beam of Fermilab, with beam power $0.7 \mathrm{MW}$ and $120 \mathrm{GeV}$ proton energy corresponding to $6 \times 10^{20}$ POT per year. This $v_{\mu}$ beam is detected by the ND of mass 280 ton at Fermilab site and the oscillated neutrino beam is observed by 14 kton far detector located near Ash River. We assume 45\% (100\%) signal efficiencies for both electron (muon) neutrino and anti-neutrino signals. 
Table 1 The values of oscillation parameters that we consider in our analysis [63]

\begin{tabular}{lll}
\hline Parameter & True value & Marginalization range \\
\hline $\sin ^{2} \theta_{12}$ & 0.310 & Not marginalized \\
$\sin ^{2} \theta_{13}$ & 0.0224 & Not marginalized \\
$\sin ^{2} \theta_{23}$ & 0.5 & {$[0.4,0.6]$} \\
$\delta_{C P}$ & $-\pi / 2$ & {$[-\pi, \pi]$} \\
$\Delta m_{21}^{2}$ & $7.39 \times 10^{-5} \mathrm{eV}^{2}$ & Not marginalized \\
$\Delta m_{31}^{2}$ & $2.5 \times 10^{-3} \mathrm{eV}^{2}$ & {$[2.36,2.64] \times 10^{-3} \mathrm{eV}^{2}$} \\
\hline
\end{tabular}

The background efficiencies for mis-identified muons (antimuons) at the detector as $0.83 \%(0.22 \%)$. The neutral current background efficiency for muon neutrino (antineutrino) is $2 \%(3 \%)$. The background contribution coming from the existence of electron neutrino (anti-neutrino) in the beam, so called intrinsic beam contamination is about $26 \%(18 \%)$. Apart from these, we assume that $5 \%$ uncertainty on signal normalization and $10 \%$ on background normalization. The auxiliary files and experimental specification of $\mathrm{NO} v \mathrm{~A}$ experiment that we use for the analysis is taken from [58].

T2K (Tokai to Kamioka) experiment is making use of muon neutrino/anti-neutrino beam produced at Tokai which is directed towards the detector of fiducial mass $22.5 \mathrm{kt} \mathrm{kept}$ $295 \mathrm{~km}$ far away at Kamioka [59]. The detector is kept $2.5^{\circ}$ off-axial to the neutrino beam axis so that neutrino flux peaks around $0.6 \mathrm{GeV}$. To simulate $\mathrm{T} 2 \mathrm{~K}$ experiment, we consider the proton beam power of $750 \mathrm{~kW}$ and with proton energy of $30 \mathrm{GeV}$ which corresponds to a total exposure of $7.8 \times 10^{21}$ protons on target (POT) with 1:1 ratio of neutrino to antineutrino modes. We match the signal and back-ground event rates as given in the latest publication of the T2K collaboration [60]. We consider an uncorrelated 5\% normalization error on signal and $10 \%$ normalization error on background for both the appearance and disappearance channels as given in reference [60] for both the neutrino and anti-neutrino. We use the Preliminary Earth Reference Matter (PREM) profile to calculate line-averaged constant Earth matter density $\left(\rho_{\mathrm{avg}}=2.8 \mathrm{~g} / \mathrm{cm}^{3}\right)$ for both NOvA and T2K experiments.

We use GLoBES software package along with snu plugin $[61,62]$ to simulate the experiments. The implementation of LIV in neutrino oscillation scenario has been done by modifying the snu code in accordance with the Lorentz violating Hamiltonian (7). We use the values of standard three flavor oscillation parameters as given in Table 1 and consider one LIV parameter at a time, while setting all other parameters to zero unless otherwise mentioned. As mentioned before, we have considered only the isotropic CPT violating parameters $\left(a_{\alpha \beta}\right)$ for our analysis. The values of the LIV parameters considered in our analysis are: $\left|a_{e \mu}\right|=\left|a_{\mu \tau}\right|=\left|a_{e \tau}\right|=2 \times 10^{-23} \mathrm{GeV}$ and $\left|a_{e e}\right|=$ $\left|a_{\mu \mu}\right|=\left|a_{\tau \tau}\right|=1 \times 10^{-22} \mathrm{GeV}$.

\section{Effect of LIV parameters on $v_{\mu} \rightarrow v_{e}$ and $v_{\mu} \rightarrow v_{\mu}$ oscillation channels}

In this section, we discuss the effect of LIV parameters $a_{\alpha \beta}=\left|a_{\alpha \beta}\right| e^{i \phi_{\alpha \beta}},\left(\phi_{\alpha \beta}=0\right.$, for $\left.\alpha=\beta\right)$, on $v_{\mu} \rightarrow v_{e}$ oscillation channel, as the long-baseline experiments are mainly looking at this oscillation channel. The evolution equation for a neutrino state $|\nu\rangle=\left(\left|v_{e}\right\rangle,\left|v_{\mu}\right\rangle,\left|v_{\tau}\right\rangle\right)^{T}$, travelling a distance $x$, can be expressed as

$i \frac{d}{d x}|v\rangle=H|v\rangle$

where $H$ is the effective Hamiltonian given in Eq. (7). Then the oscillation probability for the transition $v_{\alpha} \rightarrow v_{\beta}$, after travelling a distance $L$ can be obtained as is

$P_{\alpha \beta}=\left|\left\langle v_{\beta} \mid v_{\alpha}(L)\right\rangle\right|^{2}=\left|\left\langle v_{\beta}\left|e^{-i H L}\right| v_{\alpha}\right\rangle\right|^{2}$.

Neglecting higher order terms, the oscillation probability for $v_{\mu} \rightarrow v_{e}$ channel in the presence of LIV for NH can be expressed, which is analogous to the NSI case as [64-74],

$$
\begin{aligned}
& P_{\mu e}^{\mathrm{LIV}} \simeq x^{2} f^{2}+2 x y f g \cos \left(\Delta+\delta_{C P}\right)+y^{2} g^{2} \\
& +4 r_{A}\left|a_{e \mu}\right|\left\{x f \left[f s_{23}^{2} \cos \left(\phi_{e \mu}+\delta_{C P}\right)\right.\right. \\
& \left.+g c_{23}^{2} \cos \left(\Delta+\delta_{C P}+\phi_{e \mu}\right)\right] \\
& \left.+y g\left[g c_{23}^{2} \cos \phi_{e \mu}+f s_{23}^{2} \cos \left(\Delta-\phi_{e \mu}\right)\right]\right\} \\
& +4 r_{A}\left|a_{e \tau}\right| s_{23} c_{23}\left\{x f \left[f \cos \left(\phi_{e \tau}+\delta_{C P}\right)\right.\right. \\
& \left.-g \cos \left(\Delta+\delta_{C P}+\phi_{e \tau}\right)\right] \\
& \left.-y g\left[g \cos \phi_{e \tau}-f \cos \left(\Delta-\phi_{e \tau}\right)\right]\right\} \\
& +4 r_{A}^{2} g^{2} c_{23}^{2}\left|c_{23}\right| a_{e \mu}\left|-s_{23}\right| a_{e \tau} \|^{2} \\
& +\left.4 r_{A}^{2} f^{2} s_{23}^{2}\left|s_{23}\right| a_{e \mu}\left|+c_{23}\right| a_{e \tau}\right|^{2} \\
& +8 r_{A}^{2} f g s_{23} c_{23}\left\{c _ { 2 3 } \operatorname { c o s } \Delta \left[s_{23}\left(\left|a_{e \mu}\right|^{2}-\left|a_{e \tau}\right|^{2}\right)\right.\right. \\
& \left.+2 c_{23}\left|a_{e \mu}\right|\left|a_{e \tau}\right| \cos \left(\phi_{e \mu}-\phi_{e \tau}\right)\right] \\
& \left.-\left|a_{e \mu}\right|\left|a_{e \tau}\right| \cos \left(\Delta-\phi_{e \mu}+\phi_{e \tau}\right)\right\} \\
& +\mathcal{O}\left(s_{13}^{2} a, s_{13} a^{2}, a^{3}\right),
\end{aligned}
$$


where

$$
\begin{gathered}
x=2 s_{13} s_{23}, \quad y=2 r s_{12} c_{12} c_{23}, \quad r=\left|\Delta m_{21}^{2} / \Delta m_{31}^{2}\right|, \\
\Delta=\frac{\Delta m_{31}^{2} L}{4 E}, \quad V_{C C}=\sqrt{2} G_{F} N_{e} \\
f=\frac{\sin \left[\Delta\left(1-r_{A}\left(V_{C C}+a_{e e}\right)\right)\right]}{1-r_{A}\left(V_{C C}+a_{e e}\right)}, \\
g=\frac{\sin \left[\Delta r_{A}\left(V_{C C}+a_{e e}\right)\right]}{r_{A}\left(V_{C C}+a_{e e}\right)}, \quad r_{A}=\frac{2 E}{\Delta m_{31}^{2}},
\end{gathered}
$$

and $s_{i j}=\sin \theta_{i j}, c_{i j}=\cos \theta_{i j}$. The antineutrino probability $P_{\bar{\mu} \bar{e}}^{\mathrm{LIV}}$ can be obtained from (17) by replacing $V_{C C} \rightarrow-V_{C C}$, $\delta_{C P} \rightarrow-\delta_{C P}$ and $a_{\alpha \beta} \rightarrow-a_{\alpha \beta}^{*}$. Similar expression for inverse hierarchy can be obtained by substituting $\Delta m_{31}^{2} \rightarrow$ $-\Delta m_{31}^{2}$, i,e., $\Delta \rightarrow-\Delta$ and $r_{A} \rightarrow-r_{A}$. One can notice from Eq. (17), that only the LIV parameters $a_{e e}, a_{e \mu}$ and $a_{e \tau}$ contribute to appearance probability expression at leading order and the rest of the parameters appear only on sub-leading terms. Since Eq. (17) is valid only for small non-diagonal LIV parameter $a_{\alpha \beta}$, in our simulations the oscillation probabilities are evaluated using Eq. (16) without any such approximation, by modifying the neutrino oscillation probability function inside snu.c and implementing the Lorentz violating Hamiltonian (7).

The expression for the survival probability for the transition $v_{\mu} \rightarrow v_{\mu}$, up to $\mathcal{O}\left(r, s_{13}, a_{\alpha \beta}\right)$ is [66],

$$
\begin{aligned}
& P_{\mu \mu}^{\mathrm{LIV}} \simeq 1-\sin ^{2} 2 \theta_{23} \sin ^{2} \Delta \\
& \quad-\left|a_{\mu \tau}\right| \cos \phi_{\mu \tau} \sin 2 \theta_{23} \\
& \quad \times\left[\left(2 r_{A} \Delta\right) \sin ^{2} 2 \theta_{23} \sin 2 \Delta+4 \cos ^{2} 2 \theta_{23} r_{A} \sin ^{2} \Delta\right] \\
& +\left(\left|a_{\mu \mu}\right|-\left|a_{\tau \tau}\right|\right) \sin ^{2} 2 \theta_{23} \cos 2 \theta_{23}\left[\left(r_{A} \Delta\right) \sin 2 \Delta\right. \\
& \left.-2 r_{A} \sin ^{2} \Delta\right] .
\end{aligned}
$$

It is important to observe from the survival probability expression (19) that, the LIV parameters involved in $v_{\mu} \rightarrow v_{e}$ transitions do not take part in $v_{\mu} \rightarrow v_{\mu}$ channel. This probability depends only on the new parameters $a_{\mu \mu},\left|a_{\mu \tau}\right|, \phi_{\mu \tau}$ and $a_{\tau \tau}$.

The effect of LIV parameters on $v_{\mu} \rightarrow v_{e}$ channel for NO $v$ A experiment is displayed in Fig. 1. The left panel of the figure shows how the oscillation probability gets modified in presence of LIV, the absolute difference of standard case from Lorentz violating case (in \%) is shown in the middle panel and the relative change of the probability $\frac{\left|P_{\alpha \beta}^{\mathrm{LIV}}-P_{\alpha \beta}^{S M}\right|}{P_{\alpha \beta}^{S M}}$ is shown in the right panel of the figure. In each plot, the black curve corresponds to oscillation probability in the standard three flavor oscillation paradigm and red (blue) dotted curve corresponds to the oscillation probability in presence of LIV parameters with positive (negative) value. From Fig. 1, it is clear that all the three $a_{e \mu}, a_{e \tau}$ and $a_{e e}$ LIV parameters have significant impact on the oscillation probability. It should be further noted that the parameters $a_{e \tau}$ and $a_{e \mu}$ have impact on the amplitude of oscillation and $a_{e e}$ is affecting to phase of the oscillation, which can be seen from the Eq. (17). It should be noted from the figure that positive and negative values for LIV parameter $a_{e \tau}$, shift the probabilities in opposite direction of the standard probability curve, while the case of $a_{e \mu}$ is just opposite to that of $a_{e \tau}$ and it also creates a distortion on the probability. Also as seen from the right panel of the Fig.1, the relative change of the probability for LIV case with respect to the standard case, becomes significant towards lower energy. Furthermore, it should be inferred from the left panel of the figure that the positive and negative values of LIV parameters affect the oscillation probabilities differently. However, the result is qualitatively independent of the actual sign of LIV parameters, i.e., the spectral form of the probability is same as the standard case both for positive and negative values of LIV parameters, either it is enhanced or reduced with respect to the standard oscillation probability. Hence, one can take the $\left|a_{\alpha \beta}\right|$ for sensitivity study of the experiment in presence of LIV parameters. In Fig. 2, the effect of LIV parameters $a_{\mu \mu}, a_{\mu \tau}$, and $a_{\tau \tau}$ on $v_{\mu}$ survival probability is displayed. Analogous to the previous case, here also the effects of the parameters are noticeable; the parameter $\left|a_{\mu \tau}\right|$ significantly modifies the probability, whereas the changes due to $a_{\mu \mu}$ and $a_{\tau \tau}$ are negligibly small. In all cases, the positive or negative values of the LIV parameters are responsible for the decrease or enhancement of the oscillation probabilities. In the middle (right) panel of Fig. 2, we show the change (relative change) in oscillation probability due to the effect of LIV parameters.

\section{Sensitivity limits on the LIV parameters}

In this section, we analyse the potential of $\mathrm{T} 2 \mathrm{~K}, \mathrm{NO} v \mathrm{~A}$, and the synergy of T2K and NOvA to constrain the LIV parameters. From Eqns. (16) and (18) or from Figs. 1 and 2, it can be seen that the LIV parameters $\left|a_{e \mu}\right|$ and $\left|a_{e \tau}\right|$ along with LIV phases $\phi_{e \mu}$ and $\phi_{e \tau}$ play major role in appearance channel $\left(v_{\mu} \rightarrow v_{e}\right)$, whereas $\left|a_{\mu \tau}\right|$ and $\phi_{\mu \tau}$ influence the survival channel $\left(v_{\mu} \rightarrow v_{\mu}\right)$. In order to see their sensitivities at probability level, we define two quantities, $\Delta P_{\mu e}=\frac{\left|P_{\mu e}^{\mathrm{LIV}}-P_{\mu e}^{\mathrm{SM}}\right|}{P_{\mu e}^{\mathrm{SM}}}$ and $\Delta P_{\mu \mu}=\frac{\left|P_{\mu \mu}^{\mathrm{LIV}}-P_{\mu \mu}^{\mathrm{SM}}\right|}{P_{\mu \mu}^{\mathrm{SM}}}$, which provide the information about the relative change in probability due to the presence of LIV term from the standard case. We evaluate their values for various LIV parameters and display them in $a_{\alpha \beta}-\phi_{\alpha \beta}$ plane in Fig. 3. From the left panel of the figure, one can see that the observable $\Delta P_{\mu e}$ has maximum value at the yellow region, for $\phi_{e \mu} \approx 45^{\circ}$, if $a_{e \mu}$ is positive, whereas for negative value of $a_{e \mu}, \Delta P_{\mu e}$ is maximum for $\phi_{e \mu} \approx-135^{\circ}$. This nature of $\Delta P_{\mu e}$ can be easily understood from Eq. (16), as the appearance probability depends on sine and cosine functions 

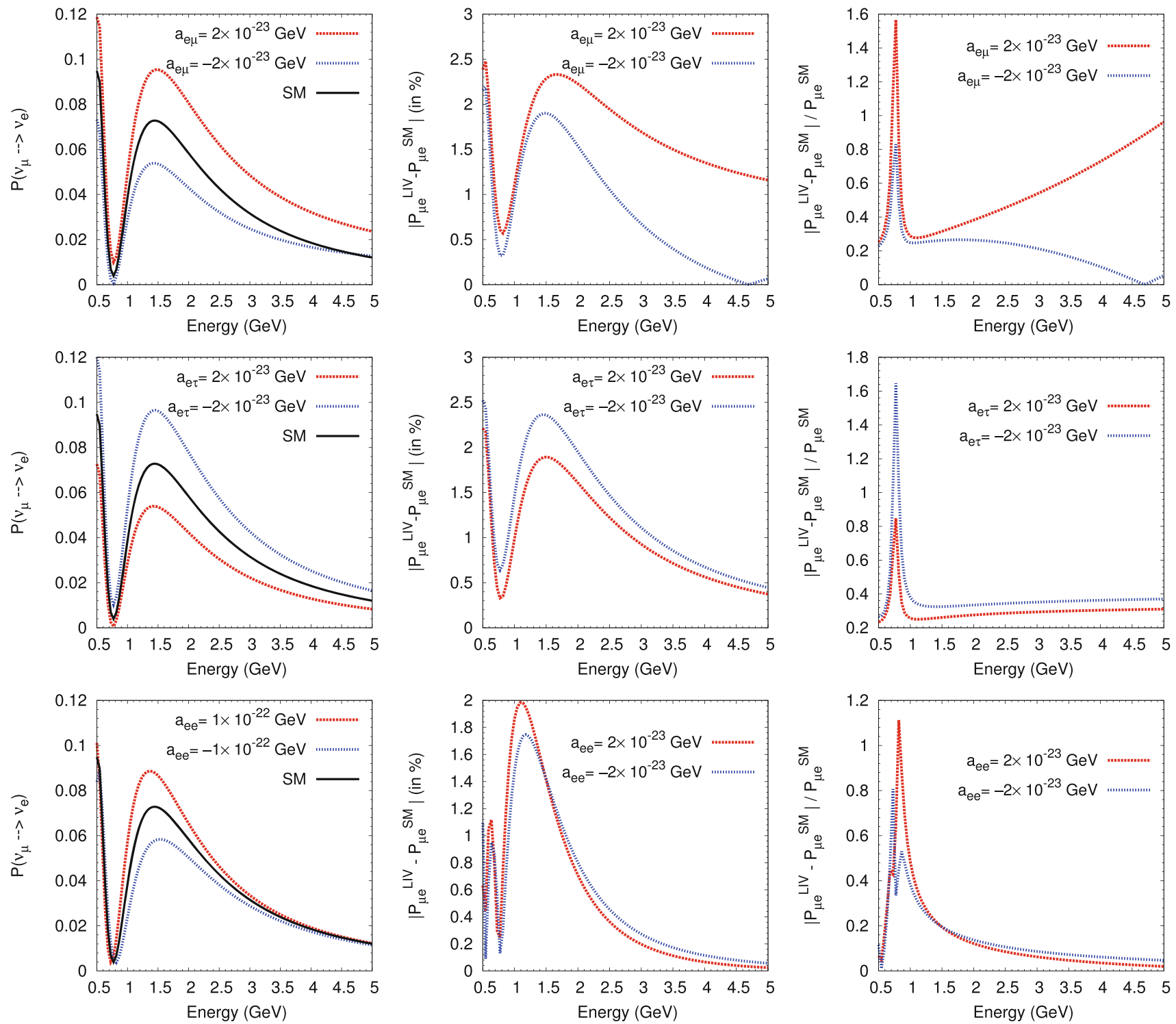

Fig. 1 The numerical oscillation probabilities for $v_{e}$ appearance channel as a function of neutrino energy for $\mathrm{NO} v \mathrm{~A}$ experiment, in presence of Lorentz violating parameters $a_{e \mu}, a_{e \tau}$ and $a_{e e}$ in the left panel. The difference in the oscillation probabilities (in \%) with and without LIV are shown in the middle panel whereas the relative change in probabilities are in the right panel of $\phi_{e \mu}$. However, the nature of $\Delta P_{\mu e}$ for $e \tau$ sector is quite different from that of $e \mu$ sector, even-though the appearance probability depends upon sine and cosine functions of $\phi_{e \tau}$. This is due to the opposite sign on $\left|a_{e \mu}\right|$ and $\left|a_{e \tau}\right|$ dependent terms in oscillation probability. As the LIV parameter $\left|a_{\mu \tau}\right|$ mainly appears on the survival channel, we calculate $\Delta P_{\mu \mu}$ which has cosine dependence on $\phi_{\mu \tau}$ and display it in the right panel of the figure.

Next, we analyze the potential of $\mathrm{T} 2 \mathrm{~K}, \mathrm{NO} v \mathrm{~A}$, and the synergy of T2K and NOvA to constrain the various LIV parameters, which are shown in Fig. 4. In order to obtain these values, we compare the true event spectra which are generated in the standard three flavor oscillation paradigm with the test event spectra which are simulated by including one LIV parameter at a time and show the marginalized sensitivities as a function of the LIV parameters, $\left|a_{\alpha \beta}\right|$. The values of $\Delta \chi_{\alpha \beta}^{2}$ are evaluated using the standard rules as described in GLoBES and the details are presented in the Appendix. From the figure, we can see that the sensitivities on LIV parameters obtained from $\mathrm{T} 2 \mathrm{~K}$ are much weaker than $\mathrm{NO} \nu \mathrm{A}$ and the synergy of T2K and NOvA can improve the sensitivities on these parameters. For a direct comparison, we give the sensitivity limits on each LIV parameter (in GeV) at $2 \sigma$ C.L. in Table 2.

All these limits are slightly weaker than the bounds obtained from Super-Kamiokande Collaboration (14). 

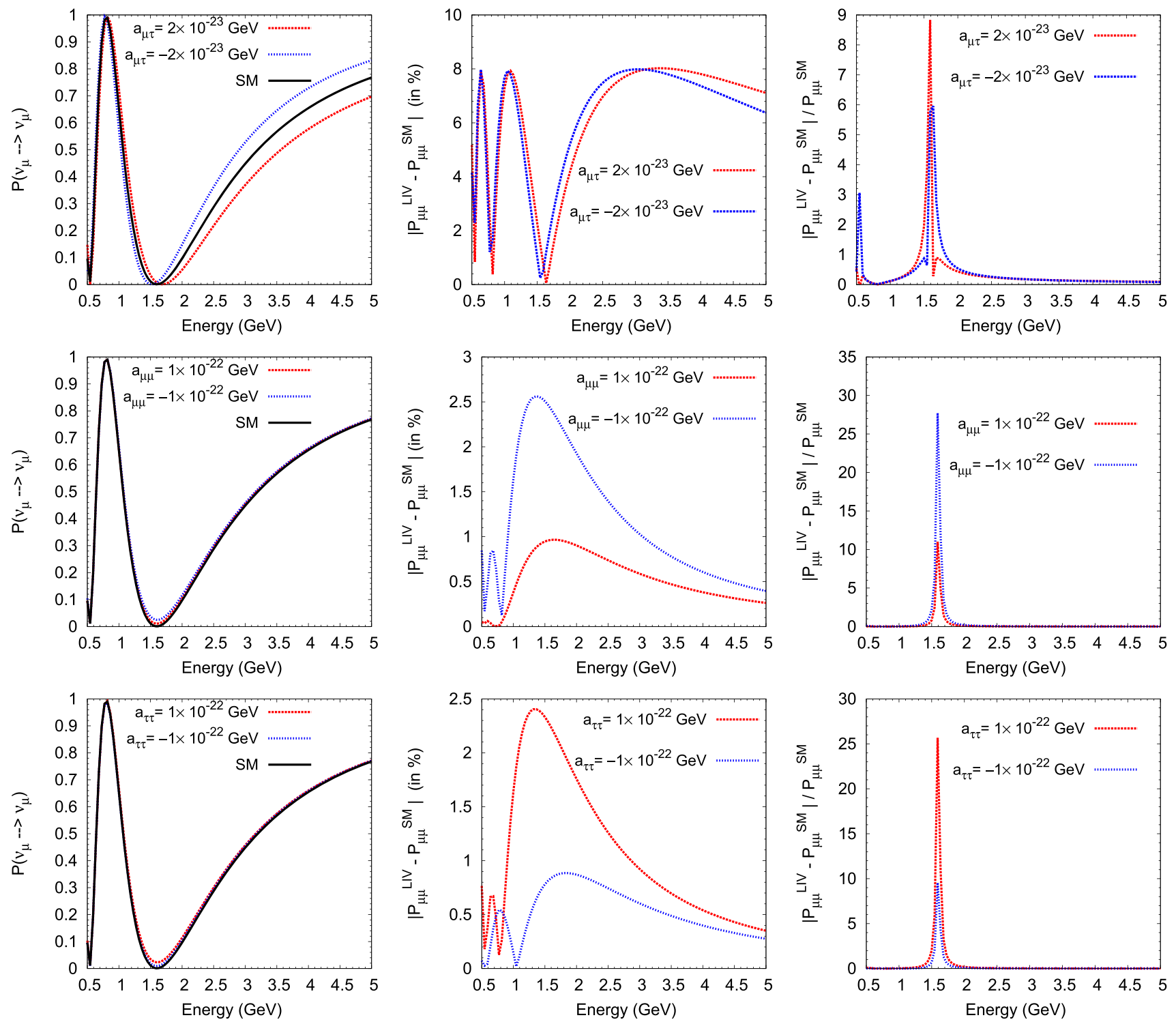

Fig. 2 Same as Fig. 1 for the $v_{\mu}$ survival probabilities as a function of neutrino energy in presence of $a_{\mu \mu}, a_{\mu \tau}$, and $a_{\tau \tau}$ LIV parameters for NO $\nu$ A experiment

\section{Effect of LIV on various sensitivities of NOvA}

In this section, we discuss the effect of LIV on the sensitivities of long-baseline experiment to determine neutrino mass ordering and $\mathrm{CP}$-violation by taking NOvA as a case of study. In addition to this, we also present the correlations between the LIV parameters and the standard oscillation parameters $\theta_{23}$ and $\delta_{C P}$.

\subsection{CP violation discovery potential}

It is well known that the determination of the $\mathrm{CP}$ violating phase $\delta_{C P}$ is one of the most challenging issues in neutrino physics today. $\mathrm{CP}$ violation in the leptonic sector may provide the key ingredient to explain the observed baryon asymmetry of the Universe through leptogenesis. In this section, we discuss how the $\mathrm{CP}$ violation sensitivity of NOvA experiment gets affected due to impact of LIV parameters. Figure 5 shows the significance with which $\mathrm{CP}$ violation, i.e. $\delta_{C P} \neq 0, \pm \pi$ can be determined for different true values of $\delta_{C P}$. For the calculation of sensitivities, we have used the oscillation parameters as mentioned in Table 1. Also, the amplitude of all the diagonal LIV parameters considered as $1 \times 10^{-22} \mathrm{GeV}$ and non-diagonal elements as $2 \times 10^{-23} \mathrm{GeV}$. The expression for the test statistics $\Delta \chi_{C P V}^{2}$, which quantifies the CP violation sensitivity is provided in the Appendix. We consider here the true hierarchy as normal, true parameters as given in Table 1 , and vary the true value for $\delta_{C P}$ in the allowed range 

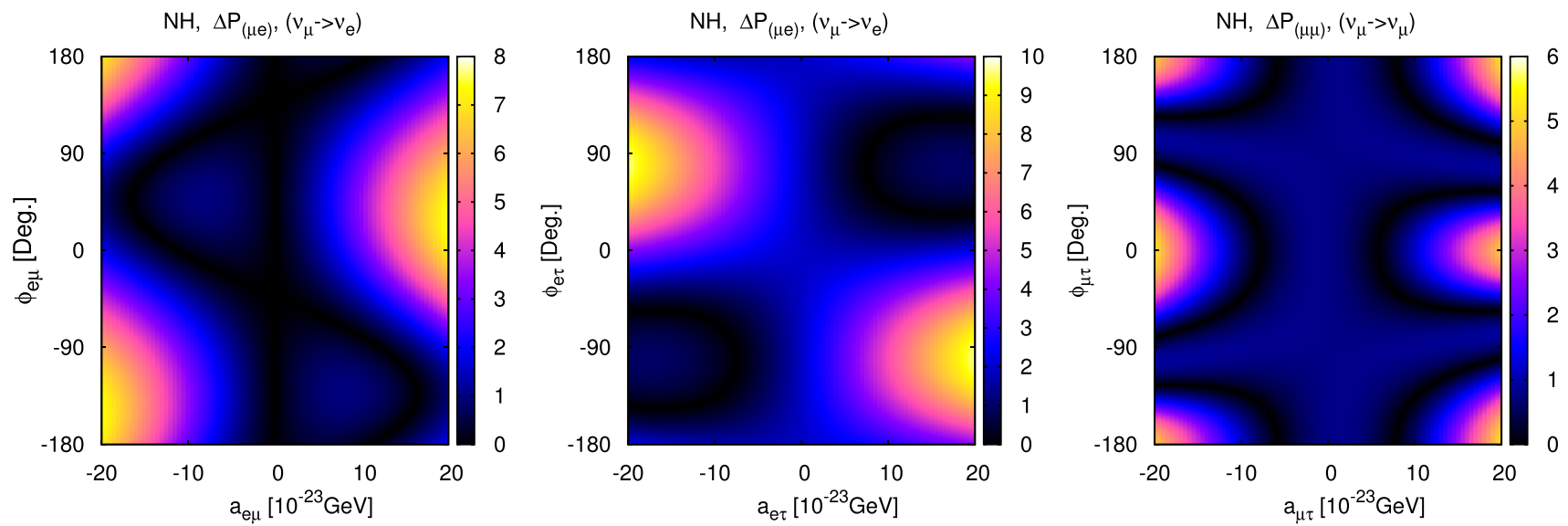

Fig. 3 Representation of $\Delta P_{\mu e}$ and $\Delta P_{\mu \mu}$ in $a_{\alpha \beta}-\phi_{\alpha \beta}$ LIV parameter space for NOvA experiment. The left (middle) panel is for the sensitivities of $\Delta P_{\mu e}$ in $a_{e \mu}-\phi_{e \mu}\left(a_{e \tau}-\phi_{e \tau}\right)$ plane and right panel is for

$\Delta P_{\mu \mu}$ in the $a_{\mu \tau}-\phi_{\mu \tau}$ plane. The color bars in right side of each plot represent the relative change of the $\Delta P_{\alpha \beta}$ in the corresponding plane

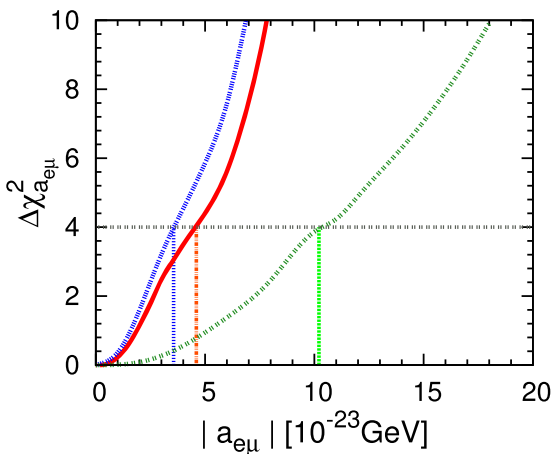

NOvA

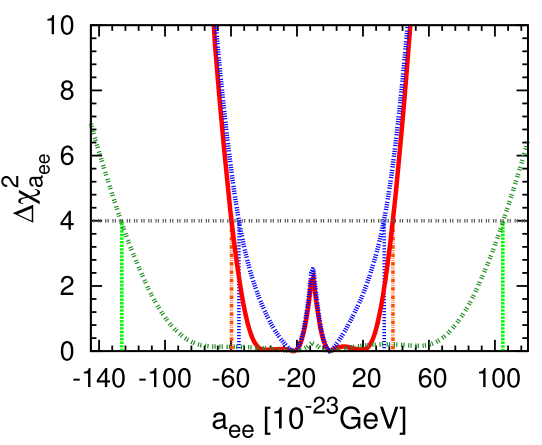

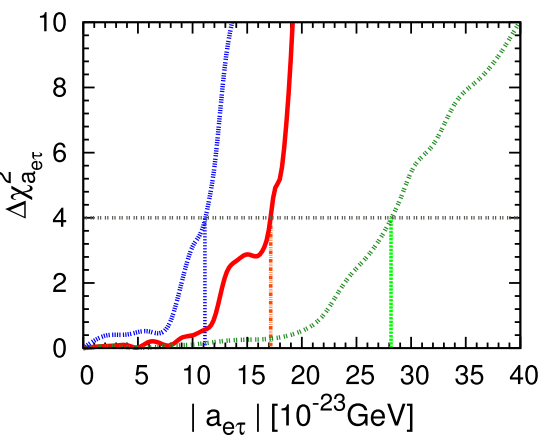

NOvA+T2K ${ }^{2} \mathrm{~K}^{2}$

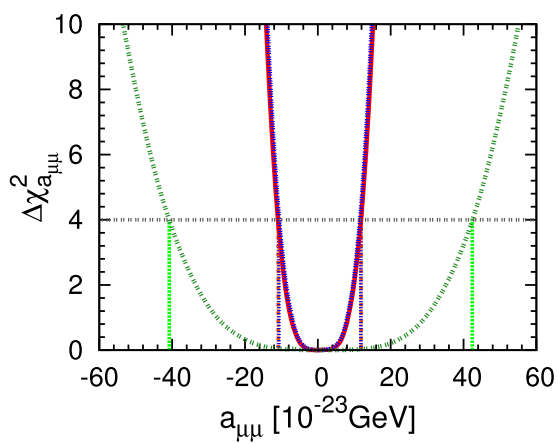

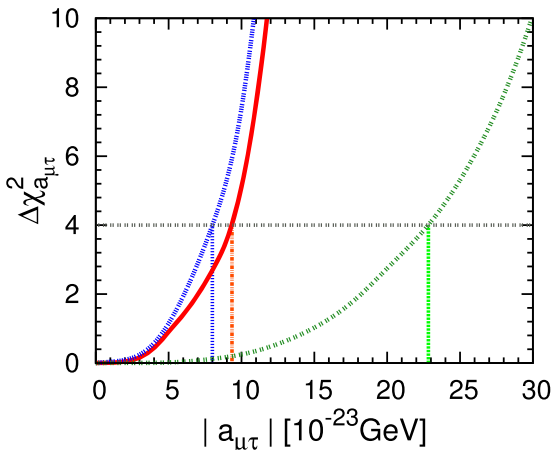

$\mathrm{T} 2 \mathrm{~K}$

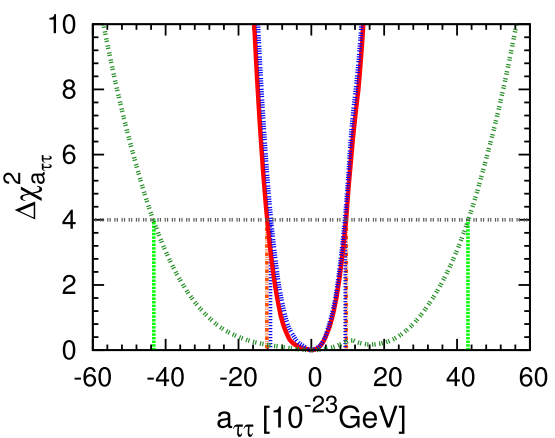

Fig. 4 The sensitivities on LIV parameters from NOvA and T2K experiments

$[-\pi, \pi]$. Also the possibility of exclusion of CP conserving phases has been shown by taking the test spectrum $\delta_{C P}$ value as $0, \pm \pi$. This exclusion sensitivity is obtained by calculating the minimum $\Delta \chi_{\min }^{2}$ after doing marginalization over both hierarchies $\mathrm{NH}$ and $\mathrm{IH}$, as well as $\Delta m_{31}^{2}$ and $\sin ^{2} \theta_{23}$ in their $3 \sigma$ ranges. The CPV sensitivity for standard case and in presence of diagonal LIV parameters is shown in the top left panel of Fig. 5. The black curve depicts the standard case, and for diagonal elements $a_{e e}, a_{\mu \mu}$ and $a_{\tau \tau}$, the corresponding plots are displayed by blue, green and red respectively. Further, we show the sensitivity in presence of non-diagonal LIV parameters in $e \mu, e \tau$, and $\mu \tau$ sectors respectively in the top right, bottom left, and bottom right panels of the same figure. As the extra phases of the non-diagonal parameters can affect the CPV sensitivity, we calculate the value of $\Delta \chi_{\min }^{2}$ for a particular value of $\delta_{C P}$ by varying the phase $\phi_{\alpha \beta}$ in its 
Table 2 The sensitivity limits on each LIV parameters (in GeV) at $2 \sigma$ C.L. from T2K, NOvA, and synergy between T2K and NOvA

\begin{tabular}{llll}
\hline LIV parameter & \multicolumn{2}{l}{ Sensitivity limi on LIV parameter } & T2K+NOvA \\
\cline { 2 - 4 } & T2K & NOvA & $<0.36 \times 10^{-22}$ \\
\hline$\left|a_{e \mu}\right|$ & $<1.02 \times 10^{-22}$ & $<0.46 \times 10^{-22}$ & $<1.08 \times 10^{-22}$ \\
$\left|a_{e \tau}\right|$ & $<2.82 \times 10^{-22}$ & $<1.71 \times 10^{-22}$ & $<0.8 \times 10^{-22}$ \\
$\left|a_{\mu \tau}\right|$ & $<2.28 \times 10^{-22}$ & $<0.93 \times 10^{-22}$ & {$[-5.52: 3.29] \times 10^{-22}$} \\
$a_{e e}$ & {$[-12.62: 10.47] \times 10^{-22}$} & {$[-5.97: 3.82] \times 10^{-22}$} & {$[-1.07: 1.18] \times 10^{-22}$} \\
$a_{\mu \mu}$ & {$[-4.09: 4.24] \times 10^{-22}$} & {$[-1.09: 1.19] \times 10^{-22}$} & {$[-1.12: 0.93] \times 10^{-22}$} \\
$a_{\tau \tau}$ & {$[-4.33: 4.3] \times 10^{-22}$} & {$[-1.22: 0.96] \times 10^{-22}$} &
\end{tabular}
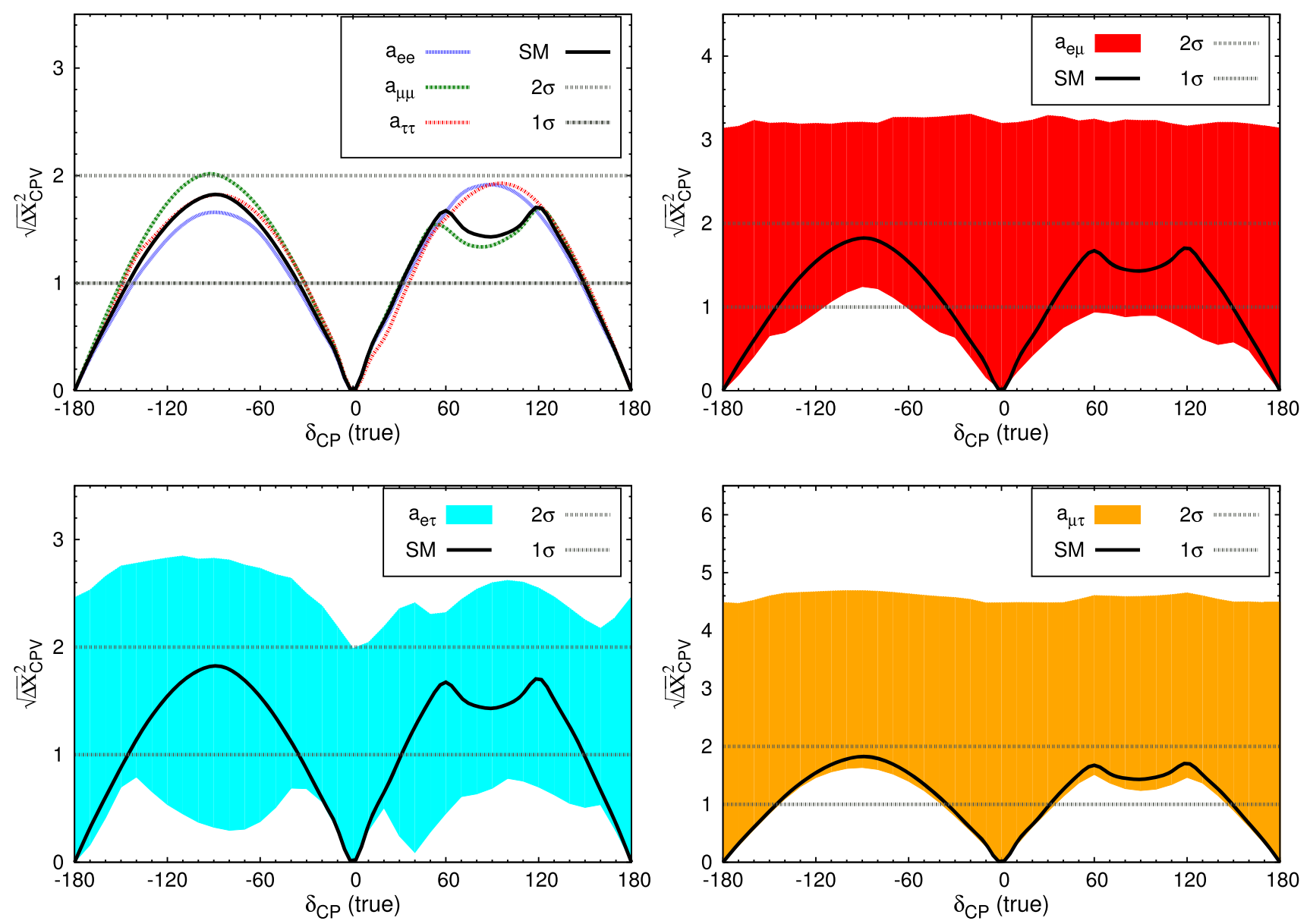

Fig. $5 \mathrm{CP}$ Violation sensitivity as a function of true values of $\delta_{C P}$ for NOvA experiment. Standard case is represented by black curve in each plot. The top-left panel is for diagonal Lorentz violating parameters

allowed range $[-\pi, \pi]$, which results in a band structure. It can be seen from figure that LIV can significantly affect the CPV discovery potential of the NOvA experiment. All the three non-diagonal LIV parameters have significant impact on CPV sensitivity. It can be seen from the figure that CPV sensitivity spans on both sides of standard case in presence of non-diagonal LIV parameters. Although there is a possibility that the sensitivity can be deteriorated in presence of LIV for and non-diagonal LIV parameters in $e \mu, e \tau$ and $\mu \tau$ sectors shown in top-right, bottom-left and bottom-right panels respectively

some particular true value of the phase of the non-diagonal parameter $\left(\phi_{\alpha \beta}\right)$, for most of the case the $\mathrm{CP}$ violation sensitivity is significantly get enhanced. Moreover, one can expect some sensitivity where there is less or no such significance for $\delta_{C P}$ regions in standard case. Further, the parameters $a_{e \mu}$ and $a_{e \tau}$ have comparatively large effect on the sensitivity with respect to to $a_{\mu \tau}$. Similar observation can also be found by considering inverted hierarchy. 

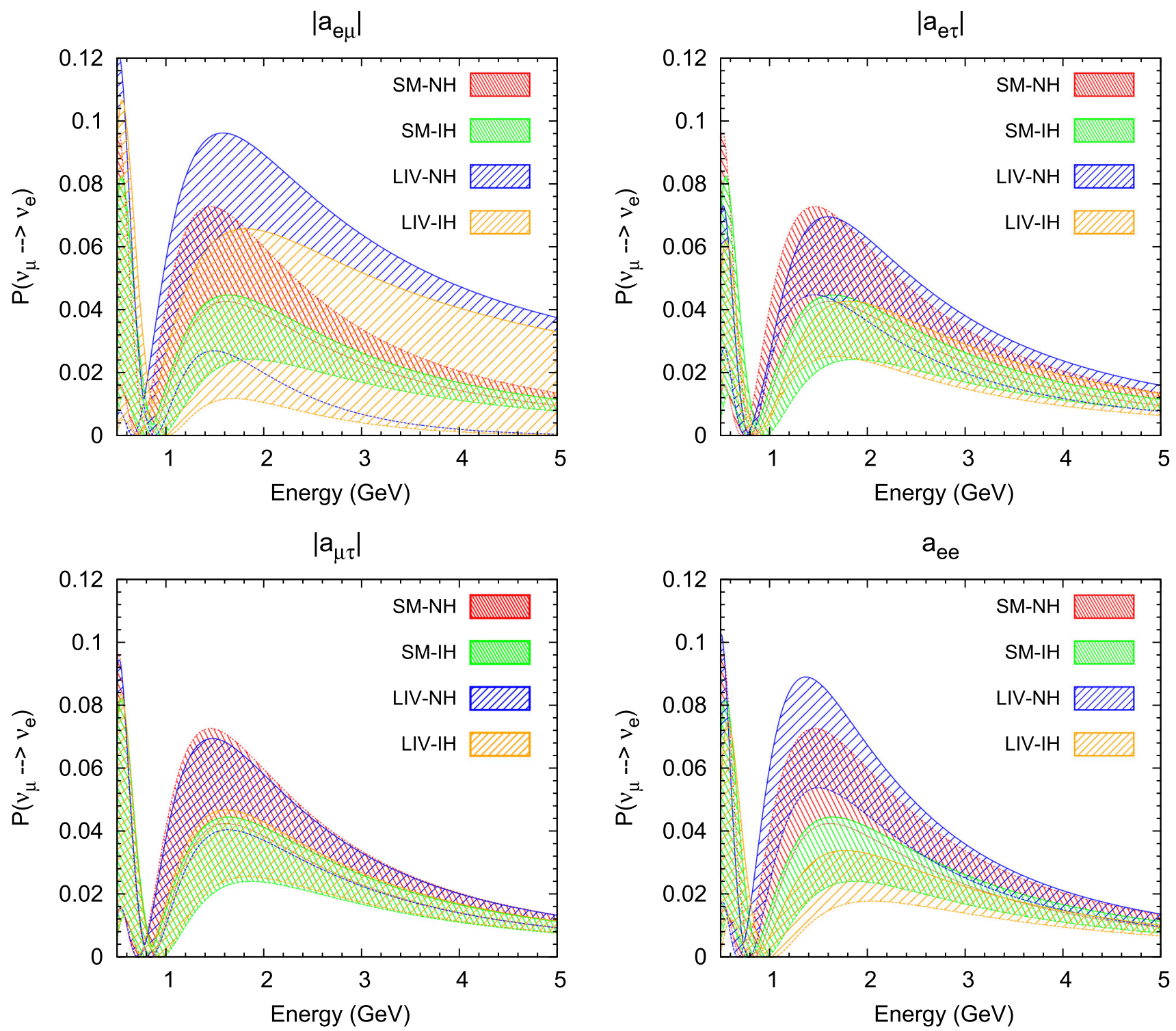

Fig. 6 The oscillation probability for NOvA experiment as a function of energy in presence of non-diagonal LIV parameters $\left|a_{e \mu}\right|,\left|a_{e \tau}\right|$ and $\left|a_{\mu \tau}\right|$ are shown in top-let, top-right and bottom-left panels respectively. The effect of diagonal LIV parameter $a_{e e}$ shown in bottom-right panel

\subsection{MH sensitivity}

Mass hierarchy determination is one of the main objectives of the long baseline experiments. It is determined by considering true hierarchy as $\mathrm{NH}(\mathrm{IH})$ and comparing it with the test hierarchy, assumed to be opposite to the true case, i.e., IH $(\mathrm{NH})$. Figure 6 shows the effect of LIV parameters on MH sensitivity at oscillation probability level. We obtain the bands by varying the $\delta_{C P}$ within its allowed range $[-\pi, \pi]$ and considering the other parameters as given in the Table 1 , and the amplitude of all the non-diagonal LIV elements as $2 \times 10^{-23} \mathrm{GeV}$ and diagonal LIV elements as $1 \times 10^{-22}$ $\mathrm{GeV}$. The red (green) band in the figure is for $\mathrm{NH}(\mathrm{IH})$ case with standard matter effect. There is some overlapped region between the two bands for some values of $\delta_{C P}$, where determination of neutrino mass ordering is difficult. The blue and orange bands represent the $\mathrm{NH}$ and $\mathrm{IH}$ case in presence of the LIV parameters respectively. It can be seen that the parameter $a_{e \mu}$ and $a_{e e}$ have significant effect on the appearance probability energy spectrum compared to other two parameters. The two bands NH and IH shifted to higher values of probability and have more overlapped regions in presence of $a_{e \mu}$. The presence of $a_{e e}$ shifted the NH band to higher values and IH band shifted to lower values of probabilities compared to standard case. Whereas the effects of $a_{e \tau}$ and $a_{\mu \tau}$ are negligibly small.

Next, we calculate the $\Delta \chi_{M H}^{2}$ by comparing true event and test event spectra which are generated for the oscilla- 


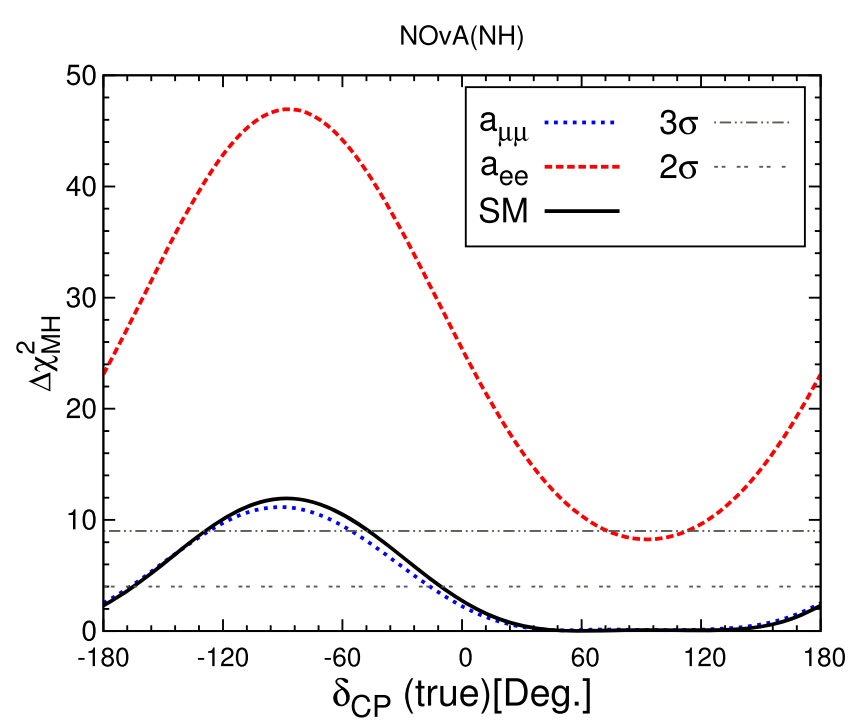

Fig. 7 Mass hierarchy sensitivity as function of $\delta_{C P}$ for NOvA experiment. Left (right) panel is for $\mathrm{NH}(\mathrm{IH})$ as true value. Black curve represents the standard matter effect case without any LIV parameter.

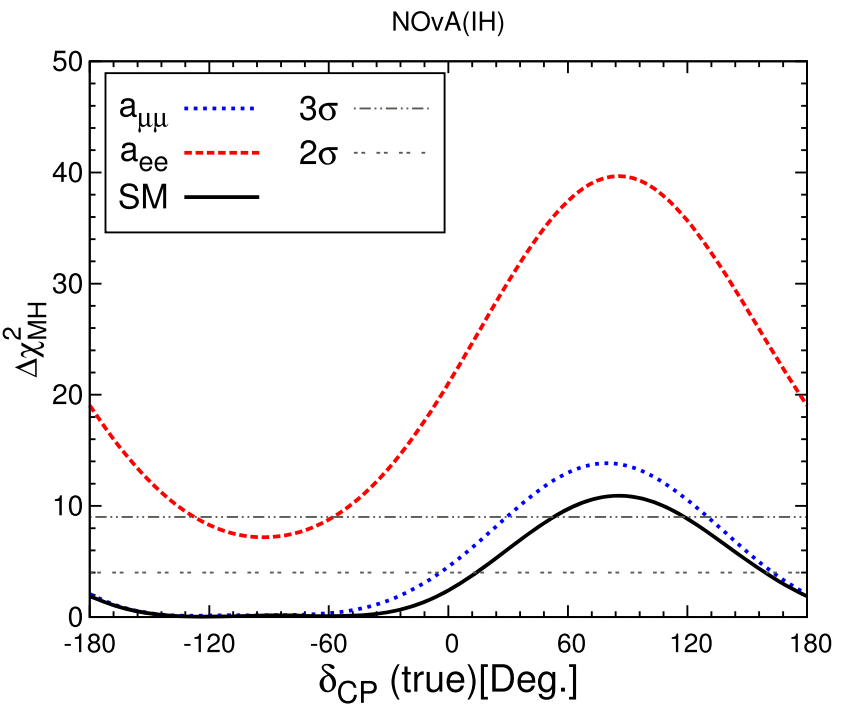

Red and blue dotted curves represent the sensitivity in the presence of diagonal parameters $a_{e e}$, and $a_{\mu \mu}$ respectively

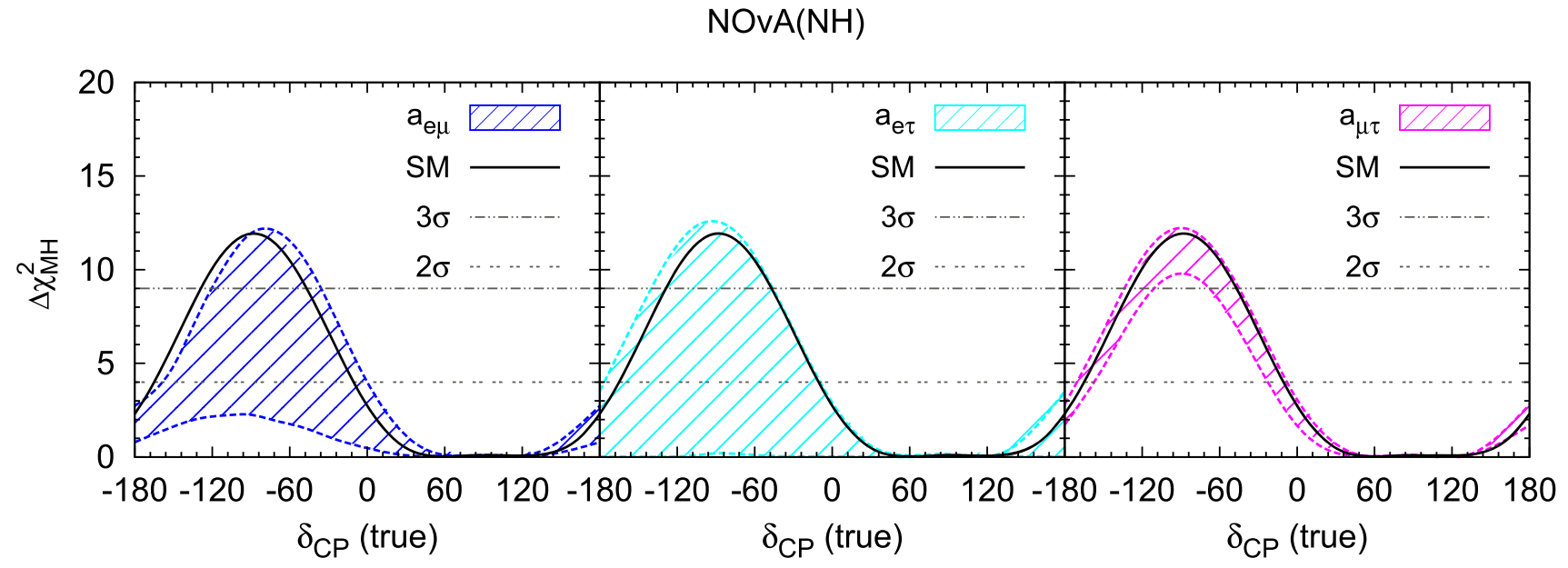

Fig. 8 Mass hierarchy sensitivity as a function of $\delta_{C P}$ for NOvA experiment in presence of $a_{\alpha \beta}$. Black curve represents the standard matter effect case without any LIV parameter. Left, middle and right panels represent the sensitivity in presence of non-diagonal parameters $a_{e \mu}, a_{e \tau}$ and $a_{\mu \tau}$ respectively

tion parameters in the Table 1 for each true value of $\delta_{C P}$. In order to get the minimum deviation or $\Delta \chi_{\min }^{2}$, we do marginalization over $\delta_{C P}, \theta_{23}$ and $\Delta m_{31}^{2}$ in their allowed regions. In Fig. 7, we show the mass hierarchy sensitivity of NOvA experiment for standard paradigm and in presence of diagonal LIV parameter. The left (right) panel of the figure corresponds to the MH sensitivity for true $\mathrm{NH}$ (IH). It can be seen from the figure that for standard matter effect case (black curve), the test hierarchy can be ruled out in upper half plane (UHP) $\left(0<\delta_{C P}<\pi\right)$ and lower half plane (LHP) $\left(-\pi<\delta_{C P}<0\right)$ for true NH and IH respectively above $2 \sigma$ C.L.. The other half plane is unfavourable for mass hierarchy determination. The parameter $a_{e e}$ is found to give significant enhancement from the standard case compared to $a_{\mu \mu}$.

It should also be emphasized that mass hierarchy can be measured precisely above $3 \sigma$ C.L. for most of the $\delta_{C P}$ region in presence of $a_{e e}$ for true value in both $\mathrm{NH}$ and $\mathrm{IH}$.

The MH sensitivity in presence non-diagonal Lorentz violating parameters $a_{\alpha \beta}$ is shown in Fig. 8. As the non-diagonal LIV parameters introduce new phases, we do marginalization over new phases in their allowed range, i.e., $[-\pi, \pi]$ while obtaining the MH sensitivity. In all the three cases, the MH sensitivity expands around the MH sensitivity in the standard three flavor framework. From the figure, it can be seen that the non-diagonal LIV parameters significantly affect the sen- 

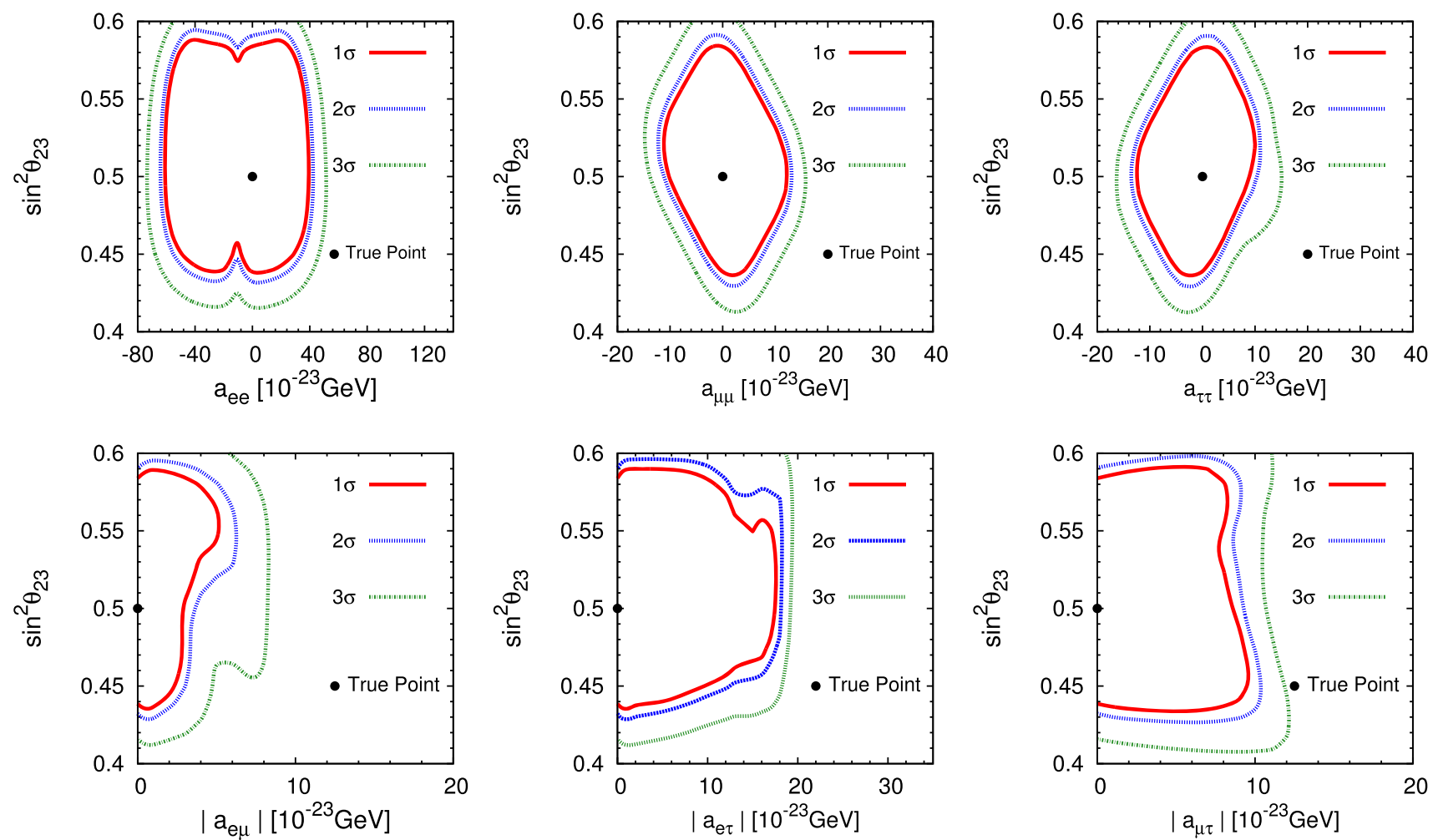

Fig. 9 Correlation between LIV parameters and $\theta_{23}$ in $\left|a_{\alpha \beta}\right|-\sin ^{2} \theta_{23}$ plane at $1 \sigma, 2 \sigma$ and $3 \sigma$ C.L. for NOvA experiment
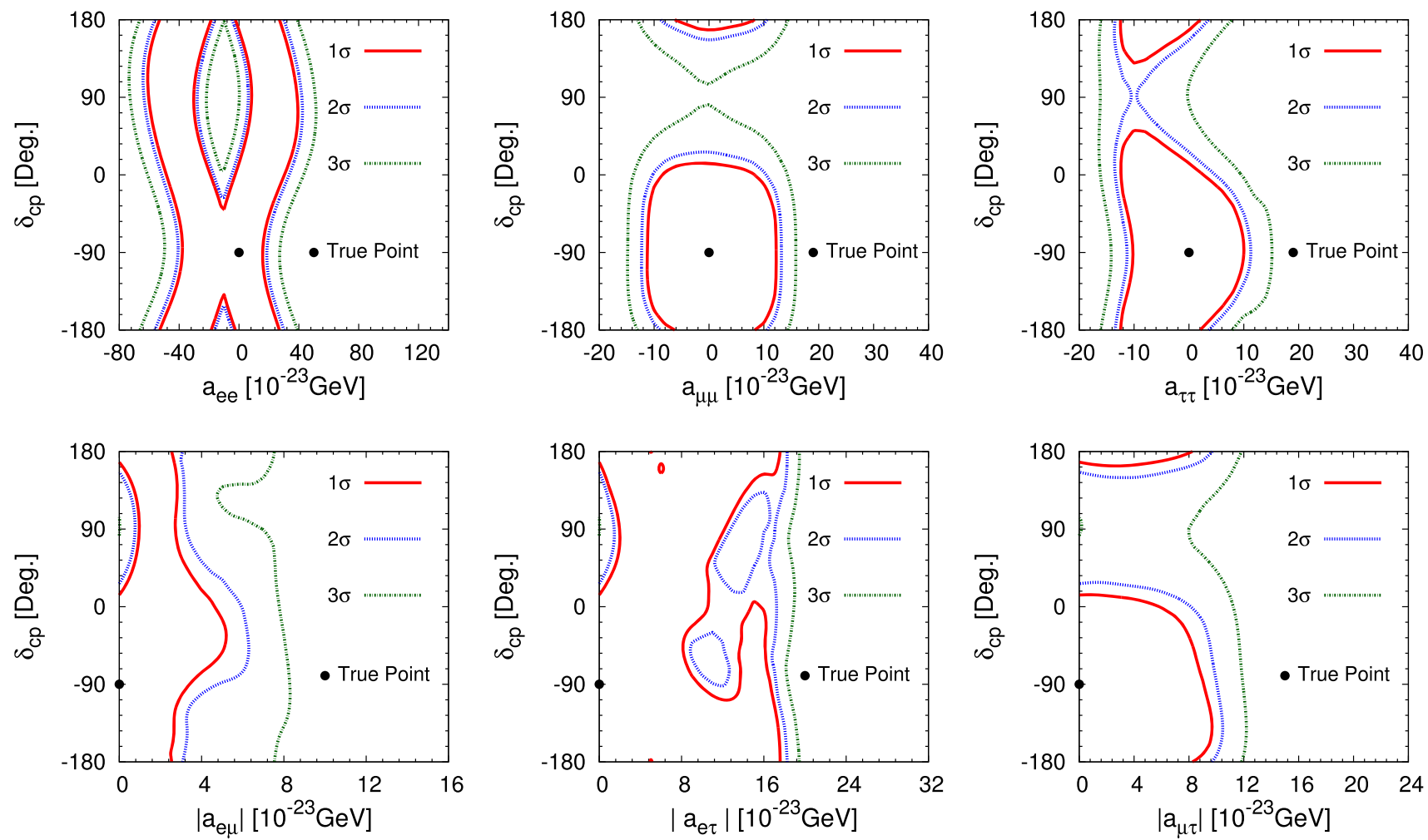

Fig. 10 Correlation between LIV parameters and $\delta_{C P}$ in $\left|a_{\alpha \beta}\right|-\delta_{C P}$ plane at $1 \sigma, 2 \sigma$ and $3 \sigma$ C.L. for NOvA experiment 
sitivity which crucially depends on the value of new phase. Similar analysis can be studied considering IH as the true hierarchy.

\subsection{Correlations between LIV parameters with $\delta_{C P}$ and $\theta_{23}$}

In this section, we show the correlation between the LIV parameters and the standard oscillation parameters $\theta_{23}$ and $\delta_{C P}$ in $\left|a_{\alpha \beta}\right|-\theta_{23}$ and $\left|a_{\alpha \beta}\right|-\delta_{C P}$ planes. Figures 9 and 10 shows the correlation for $a_{e e}, a_{\mu \mu}, a_{\tau \tau},\left|a_{e \mu}\right|,\left|a_{e \tau}\right|,\left|a_{\mu \tau}\right|$ and $\theta_{23}\left(\delta_{C P}\right)$, at $1 \sigma, 2 \sigma, 3 \sigma$ C.L. in two dimensional plane. In both figures upper (lower) panel is for $a_{e e}, a_{\mu \mu}$ and $a_{\tau \tau}\left(\left|a_{e \mu}\right|\right.$, $\left.\left|a_{e \tau}\right|,\left|a_{\mu \tau}\right|\right)$. In order to obtain these correlations, we set the true value of LIV parameters to zero and the standard oscillation parameters as given in Table 1. Further, we do marginalization over $\sin ^{2} \theta_{23}, \delta_{C P}$, and $\Delta m_{31}^{2}$ for both hierarchies. In the case of non-diagonal LIV parameters, $\left|a_{e \mu}\right|,\left|a_{e \tau}\right|,\left|a_{\mu \tau}\right|$, we also do marginalization over the additional phase $\phi_{\alpha \beta}$. From the plots it can be noticed that precise determination of $\theta_{23}$ will provide useful information about the possible interplay of LIV physics.

\section{Summary and conclusion}

It is well known that, neutrino oscillation physics has entered a precision era, and the currently running accelerator based long-baseline experiment $\mathrm{NO} v \mathrm{~A}$ is expected to shed light on the current unknown parameters in the standard oscillation framework, such as the mass ordering as well as the leptonic $\mathrm{CP}$ phase $\delta_{C P}$. However, the possible interplay of potential new physics scenarios can hinder the clean determination of these parameters. Lorentz invariance is one of the fundamental properties of space time in the standard version of relativity. Nevertheless, the possibility of small violation of this fundamental symmetry has been explored in various extensions of the SM in recent times and a variety of possible experiments for the search of such signals have been proposed over the years. In this context, the study of neutrino properties can also provide a suitable testing ground to look for the effects of LIV parameters as neutrino phenomenology is extremely rich and spans over a very wide range of energies. In this work, we have studied in detail the impact of Lorentz Invariance violating parameters on the currently running long-baseline experiments $\mathrm{T} 2 \mathrm{~K}$ and $\mathrm{NO} \nu \mathrm{A}$ and our findings are summarized below.

- Considering the effect of only one LIV parameter at a time, we have obtained the sensitivity limits on these parameters for the currently running long baseline experiments $\mathrm{T} 2 \mathrm{~K}$ and $\mathrm{NO} v \mathrm{~A}$. We found that the limits obtained from $\mathrm{T} 2 \mathrm{~K}$ are much weaker than that of $\mathrm{NO} v \mathrm{~A}$ and the synergy of $\mathrm{T} 2 \mathrm{~K}$ and $\mathrm{NO} v \mathrm{~A}$ can significantly improve these sensitivities.

- We have also explored the phenomenological consequences introduced in the neutrino oscillation physics due to the presence of Lorentz-Invariance violation on the sensitivity studies of long-baseline experiments by considering $\mathrm{NO} v \mathrm{~A}$ as a case study. We mainly focused on how the oscillation probabilities, which govern the neutrino flavor transitions, get modified in presence of different LIV parameters. In particular, we have considered the impact of the LIV parameters $\left|a_{e \mu}\right|,\left|a_{e \tau}\right|,\left|a_{\mu \tau}\right|$, $a_{e e}, a_{\mu \mu}$ and $a_{\tau \tau}$. We found that the parameters $\left|a_{e \mu}\right|$, $\left|a_{e \tau}\right|$ and $a_{e e}$ significantly affect the $v_{\mu} \rightarrow v_{e}$ transition probability $P_{\mu e}$, while the effect of $\left|a_{\mu \tau}\right|, a_{\mu \mu}, a_{\tau \tau}$ on the survival probability $P_{\mu \mu}$ is minimal. We also found that $\left|a_{e \mu}\right|$ creates a distortion on the appearance probability.

- We further investigated the impact of LIV parameters on the determination of mass hierarchy and $\mathrm{CP}$ violation discovery potential and found that the presence of LIV parameters significantly affect these sensitivities. In fact, the mass hierarchy sensitivity and CPV sensitivity are enhanced or deteriorated significantly in presence of LIV parameters as these sensitivities crucially depend on the new CP-violating phase of these parameters.

- We also obtained the correlation plots between $\sin ^{2} \theta_{23}$ and $\left|a_{\alpha \beta}\right|$ as well as between $\delta_{C P}$ and $\left|a_{\alpha \beta}\right|$. From these confidence regions, it can be ascertained that it is possible to obtain the limits on the LIV parameters once $\sin ^{2} \theta_{23}$ is precisely determined.

In conclusion, we found that $\mathrm{T} 2 \mathrm{~K}$ and $\mathrm{NO} v \mathrm{~A}$ have the potential to explore the new physics associated with Lorentz invariance violation and can provide constraints on these parameters.

Acknowledgements One of the authors (Rudra Majhi) would like to thank Department of Science \& Technology (DST) Innovation in Science Pursuit for Inspired Research (INSPIRE) for financial support. The work of RM is supported by SERB, Govt. of India through grant no. EMR/2017/001448. We acknowledge the use of CMSD HPC facility of Univ. of Hyderabad to carry out computations in this work.

Data Availability Statement This manuscript has no associated data or the data will not be deposited. [Authors' comment:All the data relevant to this publication are available in the text as well as in graphical form through the plots.]

Open Access This article is licensed under a Creative Commons Attribution 4.0 International License, which permits use, sharing, adaptation, distribution and reproduction in any medium or format, as long as you give appropriate credit to the original author(s) and the source, provide a link to the Creative Commons licence, and indicate if changes were made. The images or other third party material in this article are included in the article's Creative Commons licence, unless indicated otherwise in a credit line to the material. If material is not included in the article's Creative Commons licence and your intended use is not permitted by statutory regulation or exceeds the permit- 
ted use, you will need to obtain permission directly from the copyright holder. To view a copy of this licence, visit http://creativecomm ons.org/licenses/by/4.0/.

Funded by SCOAP ${ }^{3}$.

\section{Appendix: Details of $\chi^{2}$ analysis}

In our analysis, we have performed the $\chi^{2}$ analysis by comparing true (observed) event spectra $N_{i}^{\text {true }}$ with test (predicted) event spectra $N_{i}^{\text {test }}$, and its general form is given by

$$
\begin{aligned}
& \chi_{\text {stat }}^{2}\left(\vec{p}_{\text {true }}, \vec{p}_{\text {test }}\right) \\
& \quad=-\sum_{i \in \text { bins }} 2\left[N_{i}^{\text {test }}-N_{i}^{\text {true }}-N_{i}^{\text {true }} \ln \left(\frac{N_{i}^{\text {test }}}{N_{i}^{\text {true }}}\right)\right],
\end{aligned}
$$

where $\vec{p}$ is the array of standard neutrino oscillation parameters. However, for numerical calculation of $\chi^{2}$, we also include the systematic errors using pull method. This is usually done with the help of nuisance systematic parameters as discussed in the GLoBES manual. In presence of systematics, the predicted event spectra modify as $N_{i}^{\text {test }} \rightarrow N_{i}^{\prime}{ }^{\prime}$ test $=$ $N_{i}^{\text {test }}\left(1+\sum_{j=1}^{n} \pi_{i}^{j} \xi_{j}^{2}\right)$, where $\pi_{i}^{j}$ is the systematic error associated with signals and backgrounds and $\xi_{j}$ is the pull. Therefore, the Poissonian $\chi^{2}$ becomes

$$
\begin{aligned}
\chi^{2}\left(\vec{p}_{\text {true }}, \vec{p}_{\text {test }}, \vec{\xi}\right) & =-\min _{\vec{\xi}_{j}} \sum_{i \in \text { bins }} 2\left[N_{i}^{\prime} \text { test }-N_{i}^{\text {true }}-N_{i}^{\text {true }} \ln \left(\frac{N_{i}^{\prime} \text { test }}{N_{i}^{\text {true }}}\right)\right] \\
& +\sum_{j=1}^{n} \xi_{j}^{2} .
\end{aligned}
$$

Suppose $\vec{q}$ is the oscillation parameter in presence of Lorentz invariance violating parameters. Then the sensitivity of LIV parameter $a_{\alpha \beta}$ can be evaluated as

$$
\Delta \chi^{2}\left(a_{\alpha \beta}^{\text {test }}\right)=\chi_{\mathrm{SO}}^{2}-\chi_{\mathrm{LIV}}^{2}
$$

where $\chi_{\text {SO }}^{2}=\chi^{2}\left(\vec{p}_{\text {true }}, \vec{p}_{\text {test }}\right), \chi_{\text {LIV }}^{2}=\chi^{2}\left(\vec{p}_{\text {true }}, \vec{q}_{\text {test }}\right)$. We obtain minimum $\Delta \chi^{2}\left(a_{\alpha \beta}^{\text {test }}\right)$ by doing marginalization over $\sin ^{2} \theta_{23}, \delta m_{31}^{2}$, and $\delta_{\mathrm{CP}}$. Further, the sensitivities of current unknowns in neutrino oscillation is given by

- CPV sensitivity:

$$
\begin{aligned}
& \Delta \chi_{\mathrm{CPV}}^{2}\left(\delta_{C P}^{\mathrm{true}}\right) \\
& \quad=\min \left[\chi^{2}\left(\delta_{C P}^{\mathrm{true}}, \delta_{C P}^{\mathrm{test}}=0\right), \chi^{2}\left(\delta_{C P}^{\mathrm{true}}, \delta_{C P}^{\mathrm{test}}=\pi\right)\right] .
\end{aligned}
$$

- MH sensitivity:

$$
\begin{aligned}
& \Delta \chi_{\mathrm{MH}}^{2}=\chi_{\mathrm{NH}}^{2}-\chi_{\mathrm{IH}}^{2} \quad \text { (for true normal ordering), } \\
& \Delta \chi_{\mathrm{MH}}^{2}=\chi_{\mathrm{IH}}^{2}-\chi_{\mathrm{NH}}^{2} \quad \text { (for true inverted ordering). }
\end{aligned}
$$

Further, we obtain minimum $\chi_{\mathrm{MH}}^{2}$ by doing marginalization over the oscillation parameters $\sin ^{2} \theta_{23}, \Delta m_{31}^{2}$, and $\delta_{C P}$ in the range [0.4:0.6], [2.36:2.64] $\times 10^{-3} \mathrm{eV}^{2}$ and $\left[-180^{\circ}, 180^{\circ}\right]$ respectively, and for obtaining minimum $\chi_{\mathrm{CPV}}^{2}$ marginalization is done over the oscillation parameters $\sin ^{2} \theta_{23}$ and $\Delta m_{31}^{2}$. While including the non-diagonal Lorentz violating parameters $a_{\alpha \beta}$, we also marginalize over their corresponding phases $\phi_{\alpha \beta}$.

\section{References}

1. S. Fukuda et al., Super-Kamiokande. Phys. Rev. Lett. 86, 5651 (2001)

2. Q.R. Ahmad et al., SNO. Phys. Rev. Lett. 87, 071301 (2001)

3. Q.R. Ahmad et al., SNO. Phys. Rev. Lett. 89, 011301 (2002a)

4. Q.R. Ahmad et al., SNO. Phys. Rev. Lett. 89, 011302 (2002b)

5. Y. Fukuda et al., Super-Kamiokande. Phys. Lett. B 467, 185 (1999)

6. S. Fukuda et al., Super-Kamiokande. Phys. Rev. Lett. 85, 3999 (2000)

7. M. Apollonio et al., CHOOZ. Phys. Lett. B 420, 397 (1998)

8. F.P. An et al., Daya Bay. Phys. Rev. Lett. 108, 171803 (2012)

9. Y. Abe et al., Double Chooz. Phys. Rev. Lett. 108, 131801 (2012a)

10. J.K. Ahn et al., RENO. Phys. Rev. Lett. 108, 191802 (2012)

11. T. Araki et al., KamLAND. Phys. Rev. Lett. 94, 081801 (2005)

12. K. Eguchi et al., KamLAND. Phys. Rev. Lett. 90, 021802 (2003)

13. S. Abe et al., KamLAND. Phys. Rev. Lett. 100, 221803 (2008)

14. N. Agafonova et al., OPERA. Phys. Rev. Lett. 120, 211801 (2018)

15. K. Abe et al., T2K. Phys. Rev. Lett. 107, 041801 (2011)

16. P. Adamson et al., NOvA. Phys. Rev. Lett. 116, 151806 (2016)

17. V.A. Kostelecky, M. Mewes, Phys. Rev. D 69, 016005 (2004)

18. P. Arias, J. Gamboa, J. Lopez-Sarrion, F. Mendez, A.K. Das, Phys. Lett. B 650, 401 (2007)

19. X. Zhang, B.-Q. Ma, Phys. Rev. D 99, 043013 (2019)

20. R.G. Lang, H. Martínez-Huerta, V. de Souza, Phys. Rev. D 99, 043015 (2019)

21. B. Aharmim et al., SNO. Phys. Rev. D 98, 112013 (2018)

22. M. Mewes, Phys. Rev. D 99, 104062 (2019)

23. A. Samajdar (LIGO Scientific, Virgo), in 8th Meeting on CPT and Lorentz Symmetry (CPT'19) Bloomington, Indiana, USA, May 1216, 2019 (2019)

24. H. Martínez-Huerta, in 8th Meeting on CPT and Lorentz Symmetry (CPT'19) Bloomington, Indiana, USA, May 12-16, 2019 (2019)

25. Y. Huang, H. Li, B.-Q. Ma, Phys. Rev. D 99, 123018 (2019)

26. P. Satunin, Eur. Phys. J. C 79, 1011 (2019)

27. T. Katori, C. A. Argüelles, K. Farrag, S. Mandalia (IceCube), in 8th Meeting on CPT and Lorentz Symmetry (CPT'19) Bloomington, Indiana, USA, May 12-16, 2019 (2019)

28. B. Quinn (Muon g-2), in 8th Meeting on CPT and Lorentz Symmetry (CPT'19) Bloomington, Indiana, USA, May 12-16, 2019 (2019). http://lss.fnal.gov/archive/2019/conf/fermilab-conf-19-303-e. pdf

29. M. Tanabashi et al., Particle Data Group. Phys. Rev. D 98, 030001 (2018)

30. T. Ohlsson, S. Zhou, Nucl. Phys. B 893, 482 (2015) 
31. P. Adamson et al., MINOS. Phys. Rev. Lett. 110, 251801 (2013)

32. J.M. Carmona, J.L. Cortes, A.K. Das, J. Gamboa, F. Mendez, Mod. Phys. Lett. A 21, 883 (2006)

33. J.S. Diaz, V.A. Kostelecky, M. Mewes, Phys. Rev. D 80, 076007 (2009)

34. G. Barenboim, M. Masud, C.A. Ternes, M. Tórtola, Phys. Lett. B 788, 308 (2019)

35. B. Rebel, S. Mufson, Astropart. Phys. 48, 78 (2013)

36. J.S. Diaz, A. Kostelecky, Phys. Rev. D 85, 016013 (2012)

37. V. Antonelli, L. Miramonti, M.D.C. Torri, Eur. Phys. J. C 78, 667 (2018)

38. C. A. Argüelles (IceCube), in 8th Meeting on CPT and Lorentz, Symmetry (CPT'19) Bloomington, Indiana, USA, May 12-16, 2019 (2019)

39. M.G. Aartsen et al., IceCube. Nat. Phys. 14, 961 (2018)

40. W.-M. Dai, Z.-K. Guo, R.-G. Cai, Y.-Z. Zhang, Eur. Phys. J. C 77, 386 (2017)

41. T. Katori, C. A. Argüelles, and J. Salvado, in Proceedings, 7th Meeting on CPT and Lorentz Symmetry (CPT 16): Bloomington, Indiana, USA, June 20-24, 2016 (2017), pp. 209-212

42. J.-J. Wei, X.-F. Wu, H. Gao, P. Mészáros, JCAP 1608, 031 (2016)

43. Z.-Y. Wang, R.-Y. Liu, X.-Y. Wang, Phys. Rev. Lett. 116, 151101 (2016)

44. K. Abe et al., Super-Kamiokande. Phys. Rev. D 91, 052003 (2015a)

45. A. Chatterjee, R. Gandhi, J. Singh, JHEP 06, 045 (2014)

46. T. Katori (LSND, MiniBooNE, Double Chooz), PoS ICHEP2012, 008 (2013)

47. J.S. Diaz, Symmetry 8, 105 (2016)

48. J.S. Diaz, Adv. High Energy Phys. 2014, 962410 (2014)

49. G. Barenboim, C.A. Ternes, M. Tórtola, Phys. Lett. B 780, 631 (2018)

50. S.-F. Ge, H. Murayama (2019)

51. A. Higuera, in Proceedings, 7th Meeting on CPT and Lorentz Symmetry (CPT 16): Bloomington, Indiana, USA, June 20-24, 2016 (2017), pp. 77-80

52. Y. Abe et al., Double Chooz. Phys. Rev. D 86, 112009 (2012b)

53. T. Katori, J. Spitz, in Proceedings, 6th Meeting on CPT and Lorentz, Symmetry (CPT 13): Bloomington, Indiana, USA, June 17-21, 2013 (2014), pp. 9-12
54. S. Kumar Agarwalla, M. Masud (2019)

55. A. Kostelecky, M. Mewes, Phys. Rev. D 85, 096005 (2012)

56. M.D.C. Torri, V. Antonelli, L. Miramonti, Eur. Phys. J. C 79, 808 (2019)

57. T. Ohlsson, Rept. Prog. Phys. 76, 044201 (2013)

58. S.C, K.N. Deepthi, R. Mohanta, Adv. High Energy Phys. 2016, 9139402 (2016)

59. K. Abe et al., T2K. Phys. Rev. Lett. 118, 151801 (2017)

60. K. Abe et al. (T2K), PTEP 2015, 043 C01 (2015b)

61. P. Huber, M. Lindner, W. Winter, JHEP 05, 020 (2005)

62. P. Huber, M. Lindner, T. Schwetz, W. Winter, JHEP 11, 044 (2009)

63. I. Esteban, M.C. Gonzalez-Garcia, A. Hernandez-Cabezudo, M. Maltoni, T. Schwetz, JHEP 01, 106 (2019)

64. J. Liao, D. Marfatia, K. Whisnant, Phys. Rev. D 93, 093016 (2016)

65. J. Kopp, M. Lindner, T. Ota, J. Sato, Phys. Rev. D 77, 013007 (2008)

66. A. Chatterjee, F. Kamiya, C. A. Moura, J. Yu (2018)

67. M. Esteves Chaves, D. Rossi Gratieri, O.L.G. Peres (2018)

68. K.N. Deepthi, S. Goswami, N. Nath, Phys. Rev. D 96, 075023 (2017)

69. K.N. Deepthi, S. Goswami, N. Nath, Nucl. Phys. B 936, 91 (2018)

70. U.K. Dey, N. Nath, S. Sadhukhan, Phys. Rev. D 98, 055004 (2018)

71. O. Yasuda (2007)

72. M. Masud, A. Chatterjee, P. Mehta, J. Phys. G43, 095005 (2016)

73. M. Masud, P. Mehta, Phys. Rev. D 94, 053007 (2016a)

74. M. Masud, P. Mehta, Phys. Rev. D 94, 013014 (2016b) 